\title{
Exploring Social Attitudes to Suicide Using a Predicament Questionnaire
}

\author{
Said Shahtahmasebi1,2*, Svetlin Varbanov³, Ivan Aleksandrov33, Saxby Pridmore ${ }^{4}$ \\ ${ }^{1}$ The Good Life Research Centre Trust, Christchurch, New Zealand \\ ${ }^{2}$ Community Faculty, University of Kentucky, Lexington, KY, USA \\ ${ }^{3}$ Medical University, Varna, Bulgaria \\ ${ }^{4}$ Saint Helen's Private Hospital, University of Tasmania, Tasmania, Australia \\ Email: ${ }^{\star}$ radisolevoo@gmail.com
}

How to cite this paper: Shahtahmasebi, S., Varbanov, S., Aleksandrov, I. and Pridmore, S. (2016) Exploring Social Attitudes to Suicide Using a Predicament Questionnaire. Open Journal of Social Sciences, 4, 58-71. http://dx.doi.org/10.4236/jss.2016.412006

Received: October 31, 2016

Accepted: December 19, 2016

Published: December 23, 2016

Copyright $\odot 2016$ by authors and Scientific Research Publishing Inc. This work is licensed under the Creative Commons Attribution International License (CC BY 4.0).

http://creativecommons.org/licenses/by/4.0/

\begin{abstract}
Background: It is acknowledged that adverse events can lead to suicide. Aim: To describe a questionnaire designed to measure attitudes to suicide, and the impact of particular circumstances on the thinking. This questionnaire can be used to compare individuals and groups of individuals, including those from different countries. Method: A questionnaire based on real life adversities was developed. Respondents are presented with vignettes of fictitious characters faced with a series of difficult circumstances (predicaments). Respondents are asked to decide whether or not these characters would experience suicidal thoughts, and if so, to what degree. Responses were given numeric values and totals represented the Suicidal Attitude (SA) for each responder. We also calculated Suicidal Thoughts Induction (STI) for each predicament. Answers from 647 responders were analysed using descriptive statistic, Cronbach's alpha and factor analysis. Results: Predicament related questions form a single scale with Cronbach's alpha 0.878. Psychometric characteristics suggest the measurement of a single construct related to SA. The predicaments could be ranked. No statistical difference in SA was found between males and females. The results suggest that there may be an association between social attitudes to suicide and suicide rates. Conclusion: Social attitudes link life predicaments to suicide as an inevitable outcome. High scores on the Predicament Questionnaire in a group could translate into high suicide rates in that group. This tool may have uses in suicide prevention and further research.
\end{abstract}

\section{Keywords}

Suicide Attitudes, Predicament Questionnaire, Suicide Culture

\section{Introduction}

The World Health Organization has described as a myth, the notion that all suicide is 
the result of mental disorder [1]. We need to have better insight into individuals' attitudes and opinions about suicide. There have been many attempts to develop a suicide attitude scale, however, most are designed for specific purposes, and there is no gold standard [2] [3].

A major issue is that attitudes are subjective and change over time at both individual and social levels, often due to perceptions of suicide projected through approaches to suicide prevention. We believe a scale that excludes suicide myths will be a more likely to provide insight into suicidal thinking, and thereby, prevention.

We conceptualize suicide as the response to "predicaments" - set of unpleasant circumstances from which escape options are limited [4]. One of these is untreated or unresponsive mental disorder, but others abound [5].

Predicaments are of different kinds: suffering a painful terminal illness almost certainly involves at least some different elements to that of learning of the death of a favourite celebrity. A particular kind of predicament may perhaps exist at different levels of severity: The loss of a house key would be less distressing than the loss of the house. Also, a predicament may be more difficult to tolerate if the individual bears (justifiably or otherwise) some sense of responsibility.

The level of agreement with the notion that suicide provides a legitimate escape route out of predicaments indicates the level of permissiveness toward suicide of an individual or group/society. The response of the individual to situations is governed (in part) by the characteristics of that individual, which are determined by genetics and experience. In addition, social factors are important: in this case, the level of social permissiveness toward suicide as a solution to problems. In this study we are describing a tool which measures permissiveness toward suicide. Advanced knowledge of the level of suicidal attitudes in a group can inform the process of developing efficient suicide prevention strategies. It is of interest that individuals do not react in the same way to the same predicament.

\section{Methodology}

This study was approved by the ethics committee of the Medical University of Varna, Bulgaria.

\subsection{Study Design}

This is a prospective study exploring attitudes to suicide using a series of fictional scenarios based on practical life events. Thirty-two real life adverse situations, varying in severity and complexity, were fictionalised. These are called "Predicaments" and the questionnaire is called the "Predicament Questionnaire" (PQ) (see Appendix).

The PQ was piloted as a self-administered questionnaire on the internet. For this pilot study it was deemed unwise and inappropriate to include intimate questions about personal suicidal thoughts or attempts. The only additional data items collected in this pilot study were country of residence, age, and gender. 


\subsection{Methods}

\subsubsection{Measurement Tool}

The PQ was developed with the aim of exploring inter-cultural differences in suicidality [4]. The PQ presents 32 real life predicaments. Some are in series of increasing complexity/unpleasantness, for example, in the common predicament of a relationship break-up-in the first question $(\mathrm{Q} 1)$ the fictional characters are not cohabiting-by Q3 they cohabiting and have been married for about a year. The respondent is asked "to focus on the typical responses of people in your community/culture" and to indicate whether the designated character would have suicidal thoughts. In the affirmative case, the respondent is to indicate the degree, from slight to strong, where, "Strong suicidal thoughts are those which could (but not necessarily) result in suicidal actions (fatal or nonfatal)".

\subsubsection{Measures of Suicidal Attitude (SA) and Suicidal Thoughts}

The level of agreement/disagreement with the outcome of each predicament for fictitious individuals was given a numeric value: None $=1$, Slight $=2$, Moderate $=3$, and Strong $=4$. Using this scale, the total number of points for each respondent is a measure of suicidal attitude (SA) for each individual, which takes a value between 32 (none/low suicidality) and 128 (very high suicidality).

In addition to comparing SA of individuals to predicaments (Q1-Q32), the PQ can be used to explore the propensity of separate predicaments to induce suicidal thoughts (suicidal thought induction: STI) across different individuals or groups of individuals.

\subsubsection{Sampling Issues}

This survey questionnaire was conducted over the internet and there were no formal sampling plans or framework. The aim was to collect reactions to the questionnaire and some indication of its applicability. An invitation to complete the PQ was distributed in different countries around the world. Friends and colleague placed it on Facebook pages, and invitations were offered to Police Forces, Universities, Council Members, and Clubs which made email addresses publicly available. The survey resulted in a massive 647 responses.

\subsubsection{Analysis}

For an assessment of the internal consistency and reliability of the PQ scale factor analysis was performed using SPSS19, including evaluation of sample adequacy and Cronbach's alpha. Exploratory analysis descriptive statistic and comparative analysis were also carried out using Minitab13. Results are presented and discussed.

\section{Results}

\subsection{Sample Description}

Due to resource limitation, the questionnaire could only be offered in English. There were responses from 35 countries, the majority being English speaking, including Australia, USA, UK, New Zealand, and Ireland. For the pilot study the only demographic 
details collected were age, gender. An exploratory analysis of suicidal attitude by age and gender is shown in Table 1. It can be seen that there were 264 (41\%) male and 375 (59\%) female respondents. The average SA score of males appears to be smaller than that of females, however, this difference is not statistically significant $(\mathrm{p}=0.5)$.

For both males and females, there were proportionally more adolescent (age range 15 24 ) in this sample, and the proportion of respondents decreases across the age groups as shown in Table 1. There appears to be a relationship between age and average suicidal score (chi-Sq. $=45.2$, d.f. $=20, \mathrm{p}=0.001$ ). This may be due to the age distribution of this sample, with over representation of younger age groups.

\subsection{Reliability Analysis}

Cronbach's alpha was calculated for the overall scale to be 0.878 , which indicates a good internal consistency for the PQ. Cronbach's alpha quantifies the degree of internal consistency (reliability) - the extent to which a set of items measures a single one-dimensional latent construct or dimension of a construct [6].

For the internet pilot study it was deemed unwise and inappropriate to include intimate questions about personal suicidal thoughts or attempts, so it is not possible to carry out formal validity analysis. However, it is possible to carry out a factor analysis to determine the number of factors, the weight of each and the cumulative variation of total scores. Prior to performing a factor analysis a Kaiser-Meyer-Olkin Measure of Sampling Adequacy (KMO) was calculated to be 0.902 and Bartlett's Test of Sphericity $10,456.680(\mathrm{p}<0.001)$, which meets the criteria to proceed with factor analysis.

The result of a factor analysis is shown in Table 2 and in the Scree plot shown in Figure 1, which confirms that no item is measuring a different parameter to the main factor.

There is no evidence of a split that may indicate sub-scales or different scales. The results show a single general factor which confirms the validity of the scale. We suggest this factor to be SA.

Furthermore, it is reassuring that the change from disagreement to agreement when moving from a basic to a more complex predicament is in the expected direction. For example, when basic predicaments become more complex (and more unpleasant for the

Table 1. A descriptive comparison of male/female's suicide score across age.

\begin{tabular}{ccccccccc}
\hline \multirow{2}{*}{ Age } & \multicolumn{3}{c}{ Females } & \multicolumn{3}{c}{ Males } \\
\cline { 2 - 8 } & $\mathrm{N}$ & Mean & S.D & Range & $\mathrm{N}$ & Mean & S.D & Range \\
\hline $15-24$ & 151 & 71.92 & 16.20 & $34-107$ & 111 & 68.81 & 14.37 & $36-97$ \\
$25-34$ & 77 & 66.74 & 15.89 & $32-105$ & 69 & 69.65 & 16.54 & $36-113$ \\
$35-44$ & 55 & 64.71 & 16.43 & $34-98$ & 32 & 64.34 & 14.42 & $38-96$ \\
$45-54$ & 47 & 63.15 & 15.82 & $32-102$ & 23 & 66.00 & 18.33 & $36-106$ \\
$55-64$ & 11 & 74.09 & 25.61 & $32-107$ & 12 & 59.58 & 13.01 & $43-84$ \\
$65+$ & 34 & 71.71 & 18.99 & $33-108$ & 17 & 69.82 & 13.02 & $42-94$ \\
\hline
\end{tabular}


Table 2. Factor analysis of PVQ; Total variance explained.

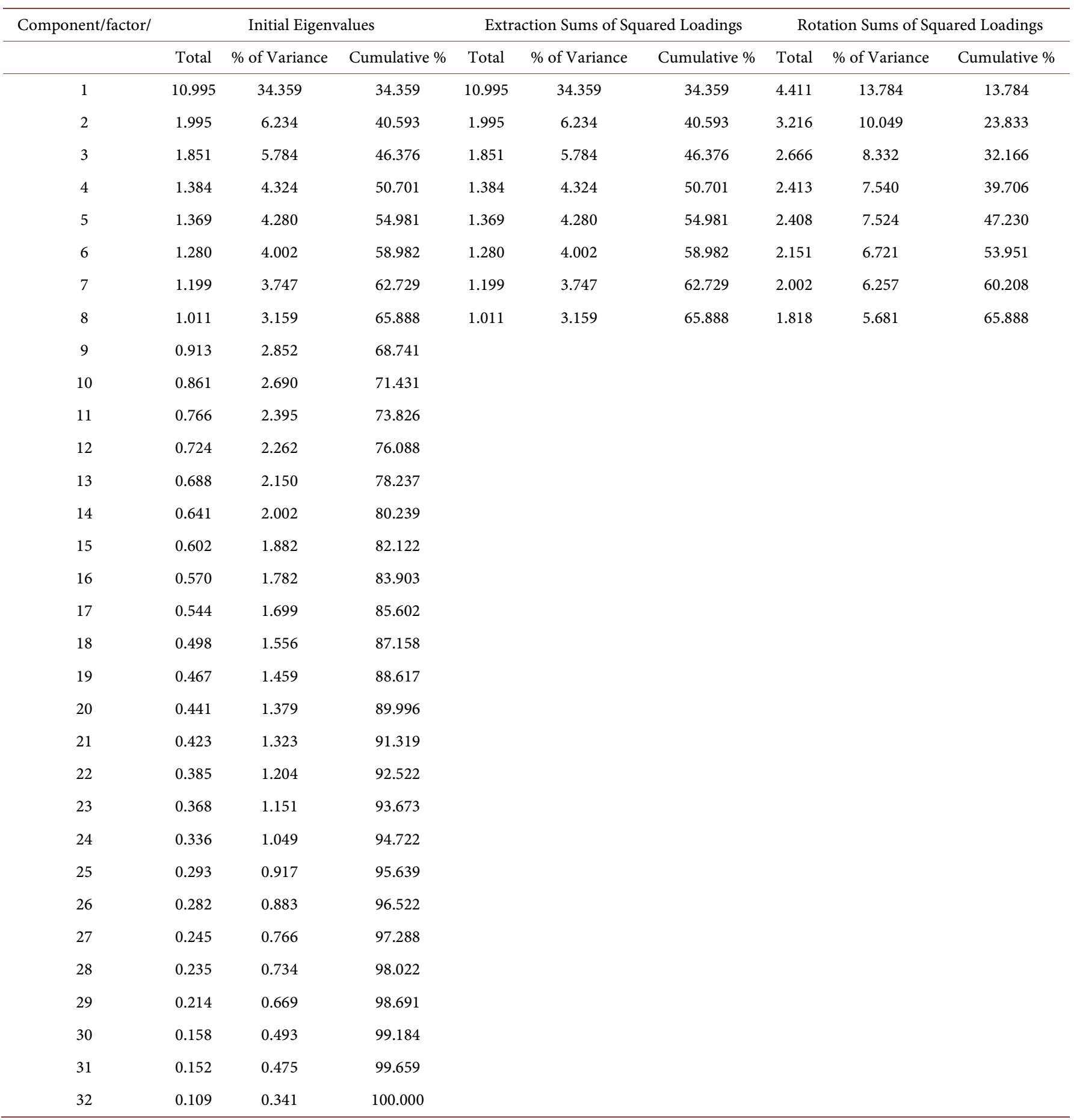

Extraction Method: Principal Component Analysis.

fictional character) proportionally more respondents changed from the opinion that there would be no suicidal thought, to the opinion that there would be suicidal thought. In Q4, a person is driving within the law and accidentally kills a pedestrian, while in Q5, the driver is drunk and speeding and kills a person on a pedestrian crossing. Re- 
sponses to these predicaments are cross-tabulated in Table 3.

\section{Discussion}

We sought to determine the amount of suicidal thought triggered by certain predicaments. The psychometric analysis of PQ presented in this paper shows that SA can be measured using PQ.

A measure that reflects attitudes to suicide at individual or social groups could be of major benefit, with implications for research and policy formation. Such a measure, in the first instance, could provide insight into the permissibility of suicide embedded in the social conscious. In other words, although suicide is undesirable and a major public health concern, the dynamics of health and social processes inevitably link it to an adverse events, i.e. "predicament".

\section{Scree Plot}

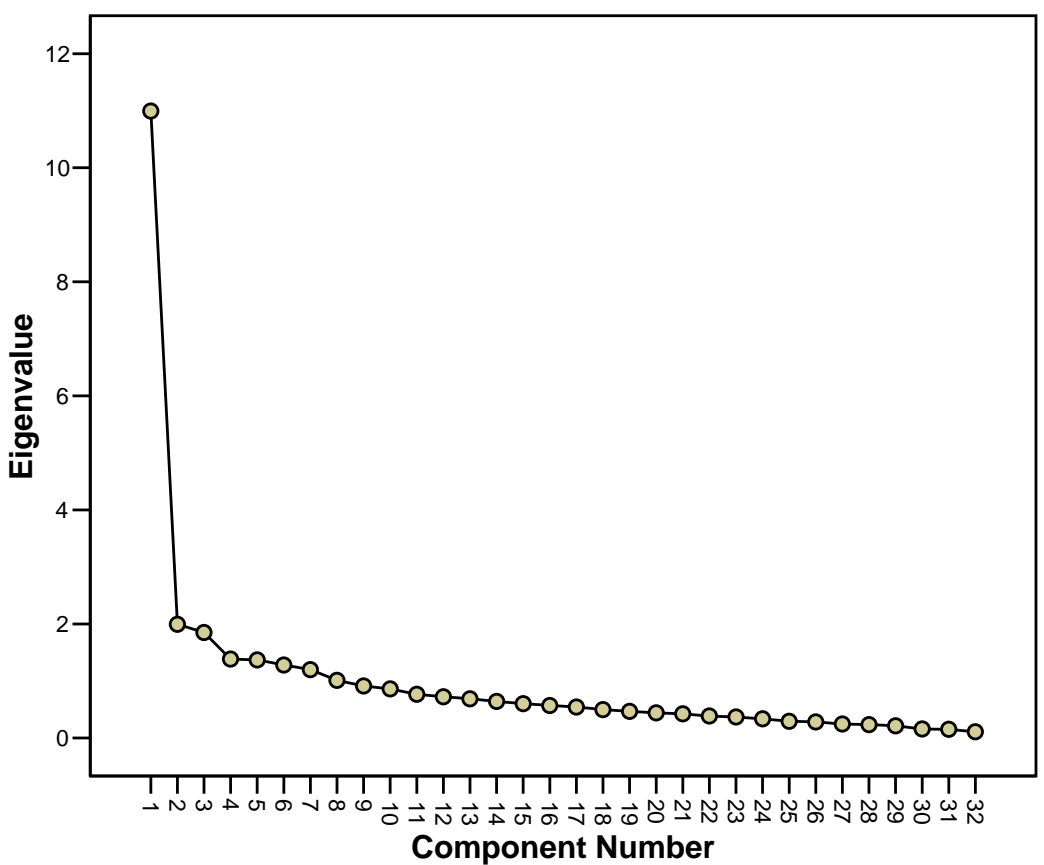

Figure 1. Scree plot analysis of PQ's components.

Table 3. Result of cross-tabulation of Q4 with Q5.

\begin{tabular}{cccccc}
\hline \multirow{2}{*}{ Q4 } & \multicolumn{5}{c}{ Q5 } \\
\cline { 2 - 6 } & No & Slight & Moderate & Strong & Total \\
\hline No & 43 & 66 & 29 & 8 & 146 \\
Slight & 6 & 72 & 97 & 30 & 205 \\
Moderate & 3 & 7 & 101 & 93 & 204 \\
Strong & 0 & 0 & 4 & 88 & 92 \\
Total & 52 & 145 & 231 & 219 & 647 \\
\hline
\end{tabular}


There are many examples of youth suicide in which the individual has been described as successful (both academically and in sport), popular, outgoing and happy, with no sign of ill-health or mental illness, yet, posthumous the individual is labelled as having suffered mental illness. For example, the following statement was made by a General Practitioner of a young individual who completed suicide who was reported as popular and happy and successful with no mental illness: "I am desperately sad we had no insight into his mental health problem and so were not able to prevent this tragedy" [7]. In other words, suicide was the outcome of adverse problems or a predicament, and mental illness had not existed. For more examples see [7] [8].

\section{Further Applications of PQ}

\section{1) Cross-national comparative study}

This questionnaire could be used to compare SA between different individuals or groups-a comparison of the SA of females and males, and across the age groups, is illustrated in Table 1.

The idea is that higher proportions of SA scores above moderate may be explored in relation to suicide rates for that group. For example, in an earlier paper [9] suicidal attitudes between English speaking countries in the sample was compared and contrasted against the latest available national suicide rates. Specifically, in our sample USA, Ireland and New Zealand scored higher on the PQ suicide attitude than UK, Canada and Australia-and the actual suicide rates are in much the same order [9].

\section{2) Suicidal Thoughts (STI)}

The 647 responses to each separate predicament were summed (STI) and ranked according to size. A statistical analysis of 647 responses giving mean and standard deviation are presented in Table 4. The five highest ranked predicaments were:

i) onset of a painful terminal illness (Q9)

ii) becoming wheelchair dependent $(\mathrm{Q} 8)$

iii) severe mental disorder causing disability (Q18)

iv) family destitute because of gambling (Q26)

v) causing a road death while intoxicated (Q5)

Thus, the predicament ranked $1(\mathrm{Q} 9), 2(\mathrm{Q} 8)$ and $3(\mathrm{Q} 18)$ all involve disability and inevitable dependency. No. 1 concerns physical (and of course, psychological) pain, and death. No. 2 predominantly concerns loss of physical independence, and No. 3 concerns mental disorder and the associated stigma. Those predicaments ranked 4 (Q26) and 5 (Q5) involve guilt/responsibility.

The importance of a notion of guilt/responsibility for the predicament is illustrated in family poverty-where destitution is the consequence of gambling, the predicament is ranked $4(\mathrm{Q} 26)$, however, when destitution is due to unemployment, it is ranked 8 (Q28). In the case of less severe family poverty, when gambling is the cause, the predicament is ranked $6(\mathrm{Q} 25)$, while when due to unemployment the predicament is ranked much lower at 16 (27).

The same kind of predicament may exist at different levels of severity, which may be 
Table 4. Mean STI score for each predicament.

\begin{tabular}{|c|c|c|c|}
\hline Predicament ${ }^{*}$ & Mean STI & Std. Deviation & $\mathrm{N}$ \\
\hline 01 & 1.38 & 0.60 & 647 \\
\hline 02 & 1.74 & 0.82 & 647 \\
\hline 03 & 2.12 & 0.96 & 647 \\
\hline 04 & 2.37 & 0.99 & 647 \\
\hline 05 & 2.95 & 0.94 & 647 \\
\hline 06 & 2.31 & 1.01 & 647 \\
\hline 07 & 2.54 & 1.01 & 647 \\
\hline 08 & 3.07 & 0.85 & 647 \\
\hline 09 & 3.26 & 0.87 & 647 \\
\hline 10 & 2.06 & 0.92 & 647 \\
\hline 11 & 2.26 & 1.00 & 647 \\
\hline 12 & 1.78 & 0.77 & 647 \\
\hline 13 & 1.94 & 0.90 & 647 \\
\hline 14 & 1.62 & 0.83 & 647 \\
\hline 15 & 1.87 & 0.96 & 647 \\
\hline 16 & 2.38 & 0.92 & 647 \\
\hline 17 & 1.39 & 0.62 & 647 \\
\hline 18 & 2.90 & 0.86 & 647 \\
\hline 19 & 1.15 & 0.43 & 647 \\
\hline 20 & 2.23 & 0.87 & 647 \\
\hline 21 & 1.13 & 0.41 & 647 \\
\hline 22 & 1.40 & 0.61 & 647 \\
\hline 23 & 1.73 & 0.84 & 647 \\
\hline 24 & 1.70 & 0.85 & 647 \\
\hline 25 & 2.53 & 0.90 & 647 \\
\hline 26 & 3.00 & 0.95 & 647 \\
\hline 27 & 2.05 & 0.87 & 647 \\
\hline 28 & 2.66 & 0.99 & 647 \\
\hline 29 & 1.89 & 1.03 & 647 \\
\hline 30 & 2.68 & 1.09 & 647 \\
\hline 31 & 2.14 & 0.98 & 647 \\
\hline 32 & 2.25 & 0.92 & 647 \\
\hline
\end{tabular}

reflected in STI responses. This is illustrated in the above paragraph, when poverty reaches destitution levels, the predicament is ranked higher than when destitution has not been reached, whether the cause is gambling or unemployment. Similarly, in the case of mental illness, when disability is reached the predicament is ranked 3 (Q18), while mild mental illness is ranked 22 (Q17). Unfortunately, this does not hold for arthritis, in this study: when the condition responds well to treatment (Q19) it is rated 9, 
while the unresponsive form (Q20) has a lower rating of 11 . We consider this an experimental error.

Social stigma of homosexuality may increase suicidal thought. When the index individual is filmed during intercourse and the film is broadcast, and the orientation is homosexual, the predicament is ranked 12 (Q7), but when heterosexual, it is ranked 20 (Q6).

There is evidence of the impact of celebrity on culture. When a celebrity dies by suicide $(\mathrm{Q} 21)$ or accident $(\mathrm{Q} 22)$ the rankings are 7 and 19. However when a life-long friend dies by accident $(\mathrm{Q} 14)$ or suicide $(\mathrm{Q} 15)$ the rankings are a much lower 30 and 31.

\section{3) Exploring dynamics of suicide}

A major advantage of the PQ is that it uses judgemental emotions, empathy, and sympathy, to explore the legitimacy of "suicide" in the public mindset. In other words, when perceived complexity of a predicament is increased, attitude to suicide appears to soften. For example, as shown in Table 4, when the circumstances of a fatal road accident are changed to drink driving, guilt is apportioned and the attitude to suicide is softened. Similarly, the severity of predicaments can trigger/stir judgemental emotions, e.g. destitution due to gambling, destitution due to unemployment, and so on [9].

These results suggest that, at least in English speaking countries, although unacceptable, suicide is given a "reluctant" legitimacy both at the individual level at the time of decision making, and at the social level, after the event. In other words, whilst the actual decision of taking one's own life is unacceptable, empathising with the individual may legitimise the option/decision to suicide. This result is reassuringly supported by respondents' comments. Out of the 647 respondents 133 chose to leave a comment. Almost all comments were from English speaking countries. One commented that suicide is rare in their culture because of religion. There were only two comments expressing personal disapproval of suicide.

\section{Conclusions}

Suicide is one of the most researched causes of human mortality. However, in spite of a huge suicide literature, we have very little understanding of the behaviour (other than mortality statistics). The literature seeks scientific evidence to explain suicide, but the science behind the evidence used to develop suicide prevention is flawed and unsustainable [7] [10] [11]. Inevitably, the World Health Organisation published a document with a section dedicated to the myths about suicide and rejecting them-including that mental illness as the cause of all suicide [1].

In this paper we have offered a new way of exploring suicide culture or attitudes, in order to better understand what might be useful in suicide prevention. To this end, the notion of adverse life events was used to develop and test the Predicament Questionnaire (PQ) which measures suicide attitude (SA). Suicide is tragic, traumatic and unacceptable, but our results suggest that in the current time, the social conscious is permissive of suicide, as it is considered to be inevitable solution to a problem.

An advantage of the PQ is that it can be included as part of any social or psychome- 
try survey to collect and test social/psychiatric theories about suicidal behaviour.

\section{Implications for Suicide Prevention}

The results show that the SA gives legitimacy to suicide as an "off the shelf" solution to a perceived predicament. The PQ measures severity by changing some circumstances of the same adverse event. However, severity, although quantifiable, is a subjective measure due to the individuals' varying threshold to problems. We must also remember that different individuals facing the same problem at same level of severity (e.g. unemployment, bereavement, ill health) will have different solutions (one or which may be suicide).

Therefore, suicide prevention programmes and action plans must target changing the social attitude to suicide. A social culture must be developed which promotes social connectivity rather than individualism, informs and depoliticise suicide, and eliminates suicide as an option [5] [7] [10] [11].

To prevent suicide, a cultural shift that eliminates the social perception of suicide as the answer to life problems and adversity is necessary [12]. This cultural shift may be achieved by the grassroots approach to suicide prevention, which reduced suicide to zero in the participating communities [5]. For example, instead of post-suicide empathy and sympathy, a social culture can change to support individuals enabling them to deal with predicaments, feeling part of the community, and feeling help is at hand and they do not have to make such tragic decisions in isolation.

\section{Limitations}

This study was piloted using the internet, and, although has achieved a large sample size, nevertheless no formal sampling method and frame was used. The results are therefore restricted to this data set only.

\section{Invitation}

The current authors are keen to hear from academics from non-English speaking regions who may be interested in using the PQ with local people with the long-term aim of a comprehensive comparison across nations/cultures.

\section{Declaration of Interest}

The authors received no funds and have no conflicts of interest.

\section{References}

[1] WHO. Preventing Suicide: A Global imperative. http://www.who.int/mental_health/suicide-prevention/world_report_2014/en/

[2] Ghasemi, P., Shaghaghi, A. and Allahverdipour, H. (2015) Measurement Scales of Suicidal Ideation and Attitudes: A Systematic Review Article. Health Promotion Perspectives, 5, 156-168. https://doi.org/10.15171/hpp.2015.019

[3] Kodaka, M., et al. (2010) A Systematic Review of Scales That Measure Attitudes toward Suicide. International Journal of Social Psychiatry, 57, 338-361. http://www.sagepub.co.uk/journalsPermissions.nav 
https://doi.org/10.1177/0020764009357399

[4] Pridmore, S. (2009) Predicament Suicide: Concept and Evidence. Australasian Psychiatry, 17, 112-116. https://doi.org/10.1080/10398560802614158

[5] Shahtahmasebi, S. (2013) De-Politicizing Youth Suicide Prevention. Frontiers in Pediatrics, 1:8. http://journal.frontiersin.org/article/10.3389/fped.2013.00008/abstract http://dx.doi.org/10.3389/fped.2013.00008

[6] Van Os, J., et al. (2002) 2-COM an Instrument to Facilitate Patient-Professional Communication in Routine Clinical Practice. Acta Psychiatrica Scandinavica, 106, 446-452.

https://doi.org/10.1034/j.1600-0447.2002.01454.x

[7] Shahtahmasebi, S. (2005) Suicides in New Zealand. Scientific World Journal, 5, 527-534. https://doi.org/10.1100/tsw.2005.74

[8] Quill, A. (2016) Teen Suicides: Something Must Change Say Devastated Parents. In: NZHerald: Auckland.

http://www.nzherald.co.nz/nz/news/article.cfm?c_id=1\&objectid=11732483

[9] Pridmore, S., et al. (2016) Social Attitudes to Suicide and Suicide Rates. Open Access Journal of Social Sciences (JSS). In Press.

[10] Hjelmeland, H., et al. (2012) Psychological Autopsy Studies as Diagnostic Tools: Are They Methodologically Flawed? Death Studies, 36, 605-626.

https://doi.org/10.1080/07481187.2011.584015

[11] Shahtahmasebi, S. (2014) Suicide Research: Problems with Interpreting Results. British Journal of Medicine and Medical Research, 5, 1147-57. https://doi.org/10.9734/BJMMR/2015/12802

[12] Pridmore, S. and Walter, G. (2013) Culture and Suicide Set Points. German Journal of Psychiatry, 16, 143-151. 


\section{Appendix}

\section{Predicament Questionnaire}

a) Gender

b) Age bracket

c) Country, and region/state/canton/prefecture

The following to be answered: No/Slight/Moderate/Strong

1. Person A had a romantic relationship of about 1 year, with person $B$, but they had not been living together. Person B ended the relationship and commenced a new relationship with a third person.

Would person A have suicidal thoughts?

2. Person A had a romantic relationship with person B of about 1 year, and they had been living together. Person $B$ ended the relationship and commenced a new relationship with a third person.

Would person A have suicidal thoughts?

3. Person A and Person B had been married for about 1 year. Person B ended the marriage and commenced a new relationship with a third person.

Would person A have suicidal thoughts?

4. Person $\mathrm{C}$ was driving below the speed limit on a suburban street. A child ran onto the road. To avoid the child, person $\mathrm{C}$ swerved and killed an adult on the other footpath.

Would person $\mathrm{C}$ have suicidal thoughts?

5. Person $C$ attended a party and got drunk. In spite of advice not to drive, and the offer of being driven home by a friend, person $\mathrm{C}$ insisted on driving. Person $\mathrm{C}$ drove above the speed limit and hit and killed a pedestrian on a pedestrian crossing. Would person $\mathrm{C}$ have suicidal thoughts?

6. Person D has heterosexual intercourse with Person Z. Without Person D's permission, this event is secretly filmed and placed on the web by a third person.

Would person D have suicidal thoughts?

7. Person D has homosexual intercourse with Person Y. Without Person D's permission, this event is secretly filmed and placed on the web by a third person.

Would person D have suicidal thoughts?

8. Person E suffers spinal injuries and will be confined to a wheelchair for life. Would person E have suicidal thoughts?

9. Person E develops a painful, terminal (will be fatal) disorder.

Would person E have suicidal thoughts?

10. Person F comes from a very high status and well educated family. Person F is convicted of stealing and jailed.

Would person $\mathrm{F}$ have suicidal thoughts?

11. Person F comes from a very high status and well educated family. Person F studies very hard, but lacks academic skills and at the end of a year at university, fails every subject.

Would person $\mathrm{F}$ have suicidal thoughts?

12. Person $G$ comes from an average family. Person $G$ is convicted of stealing and jailed. Would person $G$ have suicidal thoughts?

13. Person G comes from an average family. Person G studies very hard, but lacks aca- 
demic skills and at the end of a year at university, fails every subject.

Would person $\mathrm{G}$ have suicidal thoughts?

14. Person $\mathrm{H}$ and Person $\mathrm{X}$ lived in the same street as children and have been life-long, close friends. Person $\mathrm{H}$ is killed in a train crash.

Would person $\mathrm{X}$ have suicidal thoughts?

15. Person $\mathrm{H}$ and Person $\mathrm{X}$ lived in the same street as children and have been life-long, close friends. Person H kills him/herself by standing in the path of a train.

Would person $\mathrm{X}$ have suicidal thoughts?

16. Person J dropped a gas bottle which exploded. Person J sustained severe burns to the face and hands, which left disfiguring scars.

Would person J have suicidal thoughts?

17. Person $\mathrm{K}$ developed a mental disorder which responds well to treatment, and does not cause Person $\mathrm{K}$ to lose more than 5 working days per year.

Would person $\mathrm{K}$ have suicidal thoughts?

18. Person $\mathrm{K}$ develops a mental disorder, which does not respond well to treatment, and Person $\mathrm{K}$ is no longer able to work.

Would person $\mathrm{K}$ have suicidal thoughts?

19. Person K develops arthritis, which responds well to treatment, and does not cause Person $\mathrm{K}$ to lose more than 5 working days per year.

Would person $\mathrm{K}$ have suicidal thoughts?

20. Person K develops arthritis, which does not respond well to treatment, and Person $\mathrm{K}$ is no longer able to work.

Would person $\mathrm{K}$ have suicidal thoughts?

21. Person $\mathrm{L}$ is a great fan of Person $\mathrm{M}$, who is a popular singer, actor and talk-show celebrity. Person $\mathrm{M}$ dies when a building collapses.

Would person $\mathrm{L}$ have suicidal thoughts?

22. Person $\mathrm{L}$ is a great fan of Person $\mathrm{M}$, who is a popular singer, actor and talk-show celebrity. Person $\mathrm{M}$ dies by jumping from a building.

Would person $\mathrm{L}$ have suicidal thoughts?

23. Person N's parent has committed a serious crime. Person $\mathrm{N}$ is aware of the facts. Person $\mathrm{N}$ has been subpoenaed to appear in court and will be asked questions under oath, which will probably lead to the parent being convicted and receiving a jail sentence.

Would person $\mathrm{N}$ have suicidal thoughts?

24. Person $\mathrm{O}$ is in love with Person $\mathrm{P}$, but person O's parents want Person $\mathrm{O}$ to marry a third person, of their choosing.

Would person $O$ have suicidal thoughts?

25. Person $\mathrm{Q}$ has a serious gambling problem, has lost the family's savings and is in debt. Bills are starting to arrive which cannot be easily paid.

Would person $\mathrm{Q}$ have suicidal thoughts?

26. Person $\mathrm{Q}$ has a serious gambling problem, has lost the family's savings and is deeply in debt. Person Q's family is about to be turned out onto the street by debt collectors. Would person $\mathrm{Q}$ have suicidal thoughts?

27. Person R cannot find work and is having trouble paying the family bills. Would person $\mathrm{R}$ have suicidal thoughts?

28. Person R cannot find work and the family is destitute. Person R's family is about to 
be turned out onto the street by debt collectors.

Would person $\mathrm{R}$ have suicidal thoughts?

29. Person $S$ has a 3 year old child with terminal (will be fatal) cancer.

Would person $S$ have suicidal thoughts?

30. Person $U$ is convicted of rape and murder, and has been sentenced to life in jail without parole.

Would person $\mathrm{U}$ have suicidal thoughts?

31. Person V is the spouse of Person $U$ (the rapist-murderer in question 33).

Would person $\mathrm{V}$ have suicidal thoughts?

32. Person $\mathrm{W}$ had always been popular. However, since winning a prize, Person $\mathrm{W}$ has been subjected to a sustained, malicious web campaign, including accusations of conceit, sexual deviance and dishonest acts.

Would person $\mathrm{W}$ have suicidal thoughts?

Submit or recommend next manuscript to SCIRP and we will provide best service for you:

Accepting pre-submission inquiries through Email, Facebook, LinkedIn, Twitter, etc.

A wide selection of journals (inclusive of 9 subjects, more than 200 journals)

Providing 24-hour high-quality service

User-friendly online submission system

Fair and swift peer-review system

Efficient typesetting and proofreading procedure

Display of the result of downloads and visits, as well as the number of cited articles

Maximum dissemination of your research work

Submit your manuscript at: http://papersubmission.scirp.org/

Or contact jss@scirp.org 